Economics

\title{
Local Social Interaction and Urban Equilibria
}

available online : http://wwwfr.uni.lu/recherche/fdef/crea/publications/discussion_papers

\section{Emmanuelle Augeraud-Veron, GREThA, UMR 5113, University of Bordeaux, France}

Francisco Marhuenda, University Carlos III, Madrid, Spain

Pierre M. Picard, CREA, Université du Luxembourg

November, 2019

For editorial correspondence, please contact: crea@uni.lu

University of Luxembourg

Faculty of Law, Economics and Finance

6, Rue Richard Coudenhove-Kalergi

L-1359 Luxembourg

The opinions and results mentioned in this paper do not reflect the position of the Institution 


\title{
Local Social Interaction and Urban Equilibria
}

\author{
Augeraud Veron, E., ${ }^{*}$ Marhuenda F. ${ }^{\dagger}$ and Picard P.M. ${ }^{*}$
}

November 20, 2019

\begin{abstract}
In this paper we investigate the effect of local interaction in a simple urban economics model. Agents interact with others if and only if their interaction benefit outweights their travel cost and therefore meet others only within finite geographic windows. We show that two or more cites may co-exist at the equilibrium provided that they are sufficiently distant. For any interaction surplus function, there exists a unique spatial equilibrium on not too large city supports. The population density within a city is determined by a second order advance-delay differential equation, whose solutions are fully characterized for linear interaction surplus functions. $\mathrm{Nu}-$ merical analyses show that more localized interactions yield flatter population density and land rents over larger extents of the city support. They do not give support to the idea that multiple subcenters can be caused by small and finite geographic windows of interaction.
\end{abstract}

Keywords: social interaction, cities, spatial equilibrium.

J.E.L.: R12

\footnotetext{
${ }^{*}$ Emmanuelle Augeraud-Veron, GREThA, UMR 5113, University of Bordeaux.

${ }^{\dagger}$ Marhuenda Francisco, University Carlos III, Madrid, UC3M.

${ }^{\ddagger}$ Pierre M. Picard, University of Luxembourg, CREA. Email: pierre.picard@uni.lu.

${ }^{\S}$ We thank P. Mossay and R. Boucekkine for support and useful guidance.
} 


\section{Introduction}

The urban economic literature explains the emergence of a city through the existence of agglomeration forces. Such forces may stem from face-to-face interaction between managers (Fujita and Ogawa, 1982), technological spillover between firms (Berliant et al., 2002; Rossi-Hansberg and Lucas, 2003), social interactions between residents (Mossay and Picard, 2011 and 2019), or demand and forward linkages by firms (Mossay, Picard and Tabuchi 2017). A common simplification in this theoretical literature lies in the assumption of global interaction within the city. That is, it is assumed that, in an urban area, all managers find it attractive to meet every other manager, all firms benefit from copying a bit of every other firm's technology, residents are willing to interact with every other and, all firms find it profitable to sell and buy intermediate goods to and from every other firm.

The assumption of global interaction is however unwarranted in large urban areas. Intuitively, one does not crosses the full city extent of a large metro area for every types of service, purchase, job or social interaction. Indeed, empirical studies show that distance strongly reduces the level of socio-economic interactions. Büchel and von Ehrlich (2016) show that 50 percent of ties formed by Swiss mobile telephone users lie within a $5 \mathrm{~km}$ perimeter, which covers on average less than 1 percent of the population. Furthermore, the advent of the Internet has not much change habits: Mok et al. (2010) show that, face-to-face contact remained strongly related to short distances and with same frequency among friends and relatives between the 1970ies and 2000ies. Levy and Goldenberg (2014) show that, the probability of a social link in a sample of Facebook and email users is proportional to the inverse of the square of the distance between them.

To account for the negative effect of distance on interaction, most urban economic models combine the assumption of global interaction with decaying interaction effectiveness, typically through negative exponential/iceberg travel cost of interaction. When the benefit of interaction decreases at a constant rate with distance, it gets very small at very large distance but never nullifies, which is a rather unrealistic feature. The purpose of 
this paper is to investigate the alternative case of 'local interaction' where such benefit becomes zero at some 'finite' distance.

Towards this aim, we revisit Beckmann's (1976) linear city model with social interaction and homogeneous population. Agents have elastic demand for residential land while they benefit from interaction with each other and incur a travel cost for every interaction. Agents bid for residential land, compete with each other and with other land users (typically, farmers). In this city, the social interaction (net) benefit act as the agglomeration force and the land market as the dispersion force. In contrast with the literature, agents do not interact with too far individuals because interaction benefits are there lower than their costs. For analytical tractability, we focus on the case of linear travel costs although many results do not qualitatively hinge on such an assumption. We firstly explain the difference between local and global interaction. In particular, the spatial equilibrium includes one city under global interaction while many equilibria exist with many configurations of sufficiently distant cities under local interaction.

In this paper, we focus on local interaction and their impact on the spatial distribution of residents in a city. To the best of our knowledge, our approach contrasts to most urban studies of city structure with global interaction. Indeed, we characterize the spatial equilibrium as a fixed point of the population density function. We show that local interaction imply that the equilibrium population density function is the outcome of a second order advanced-delay differential equation. That is, the changes in population density at any urban location depend on the density at some specific distance to the left and right hand of this location. This specific distance corresponds to the interaction window' of agents, that is, the geographical support in which each agent has an incentive to make an interaction. The presence of local interaction makes the problem complex due to the solving of advanced-delay differential equations. We use the Contraction Mapping theorem to show the existence and uniqueness of a spatial equilibrium for a subset of economic parameters by for any type of interaction surplus. In the case of linear interaction surplus, the solution for population density is also found to be a piecewise trigonometric 
function of the geographic coordinates of each agent's residence.

The characterization of spatial equilibria is further complicated by the fact that land can be used for other purposes (e.g. farming) than residence. To offer a land bid above that of those other land users, residents must be content with small enough land plots. As population density is inversely related to residents' land use, this means that the equilibrium population density should be high enough within a city. The equilibrium population density therefore jumps from a positive value to zero at the city border. Checking this condition with the above piecewise trigonometric function for population density proves analytically infeasible. Our strategy is to hold that condition and analytically prove that, for generic parameter values of preferences for space, interaction benefit and travel cost, there exists a spatial equilibrium candidate. The latter are proved to be spatial equilibria only for not too large city supports. Numerical simulations then allow us to verify the existence of a unique spatial equilibrium for larger city supports.

Our study confirms that usual properties of urban equilibria apply under local interactions. Bigger population lie on larger geographical extent. However, the latter tends to become inelastic for large enough cities. Higher opportunity cost of land increases population density and attracts a larger population on a same geographical extent. Stronger preferences for residential space make cities wider. A new result specific to local interaction is that more localized interactions tend to yield flatter population density and land rents over a wider city support.

The study also sheds light on the debate about mono- and poly-centric cities. In the urban economics literature, multiple subcenters coexist under the assumptions of global interaction and heterogeneous groups of land users (managers and residents in Fujita and Ogawa, 1982; firms and residents in Berliant et al. 2002 and Rossi-Hansberg and Lucas, 2003; residents with different communities in Mossay and Picard, 2015; Blanchet et al., 2016 etc.). This paper suggests that the first assumption is not the key one. We indeed focus on the location a homogeneous group of residents and find that sufficiently distant cities emerge as spatial equilibria under local interaction. But we do not find examples 
for the emergence of several subcenters within the same city. As soon as two cities become close enough, equilibrium forces merge them into a single city with single-peaked population density profile.

Our urban model with local interaction is described by an advanced-delay differential equation, also called Mixed-type Functional Differential Equation (MFDE in the following). This is because interaction takes place within a finite window of the urban space. Delay differential equations are used in dynamic applications where delays are present. They have been used in many areas of physics and engineering (system control), life science (epidemiology, immunology, physiology, population dynamics and neural networks). They have very early been recognized as an important aspect of economics for the oscillatory and unstability properties that are created by the reaction delays of investors, governments, etc. (Frisch and Holme, 1935; James and Belz, 1938). Because of their analytical complexity, they have led to a scant amount of economic applications. In growth theory, MFDEs have been used by Boucekkine et al. (2005) who study vintage capital models in which investment are delayed. Solving the optimal control problem using Pontryagin's method yields to a state variable with delays and an adjoint variables with advances. Continuous time overlapping generations models also lead to MFDE when agents have finite lifetime (d'Albis and Augeraud, 2007). Long run properties of the dynamics are mainly studied (d'Albis et al. 2014). Even if the dynamics we encounter in this paper is given by a MFDE, its study completely differs from the one used in the previously cited papers. The dynamics is here written according to a variable of the geographical space rather than time (thus the long run properties are not what we are interested in). Moreover, our dynamics is defined on a finite interval. Basic mathematical toolkits are provided in Diekmann et al. (1995) and Mallet-Paret and Verduyn-Lunel (2001) who study MFDE on both infinite and finite intervals.

We present our urban economics model with local interactions in Section 2 and its general properties in Section 3. We then focus on the linear travel cost case in Section 4 and explain the difference between spatial equilibria local and global interactions. In 
that section, we construct the spatial equilibria and discuss simulation exercises. Section 5 concludes.

\section{The Model}

In this section we present the economic environment and define the urban spatial equilibrium. For the sake of simplicity, we focus on a one dimensional city, which is modelled by a line. We assume that the urban residential area lies on a unit-width strip. Land has an opportunity value $R_{A}>0$ given by land farming. We denote land locations on this road by $x \in \mathbb{R}$. An $M$ unit mass of agents distributes along this road according to the population density function $\lambda \in \mathcal{F}$ where $\mathcal{F}$ denotes the class of integrable functions $\mathbb{R} \rightarrow \mathbb{R}_{+}$. Agents travel along the road and benefit from social contacts with other agents. The social surplus that an agent in location $x$ derives from interacting with other agents at locations $y$ is given by the operator $S: \mathcal{F} \rightarrow \mathcal{F}$

$$
(S \lambda)(x)=\int_{\mathbb{R}} A(x-y) \lambda(y) \mathrm{d} y
$$

where $A: \mathbb{R} \rightarrow[0,1]$ represents the interaction surplus with an agent at distance $|x-y|$. We define $A(x)=\max [1-T(\tau x), 0]$ where $\tau \in \mathbb{R}_{+}$is a travel cost parameter and $T \in \mathcal{F}$ is a travel cost function that is continuous, increasing for $x>0$, nil at $x=0$ and symmetric about $x=0$. As a result, $A: \mathbb{R} \rightarrow[0,1]$ is a symmetric and nonincreasing function of distance. Because of the travel cost, interaction benefits may not balance large travel costs so that agents have "local" interactions only with their neighborhood $[x-a, x+a]$, where $T(\tau a)=1$. We call $2 a$ the interaction window size. A linear travel cost is defined as $T(\tau x)=\tau|x|$ where $\tau$ is the travel costs per distance unit so that $a=1 / \tau$. The spatial properties induced by local interactions are the main focus of the paper.

The social utility $(S \lambda)(x)$ can be interpreted in a context of certainty or uncertainty. Indeed, it can be the utility derived by an individual who plans to interact with all other agents with probability 1 . It can also be the expected utility of an individual who plans to interact with a subset of agents whom location and identity are not known at the time of 
the residence choice. Such an interpretation applied to the case of shopkeepers, sellers, as well as workers who expect to hold several jobs at different locations during their lifetime.

Agents consume a quantity $z$ of composite good and a quantity $s$ of land space. Their utility at residential location $x$ is given by

$$
U(S \lambda, s, z, x)=(S \lambda)(x)+z-\frac{\beta}{2 s}
$$

where $\beta$ reflects their preference for land use. They face a budget constraint given by $z+R(x) s \leq Y$ where $Y$ is an exogenous income and $R \in \mathcal{F}$ denotes the land rent in $x$. Because preferences are increasing in consumption, agents have incentives to spend their whole budget. By using the binding budget constraint, the utility derived in location $x$ can be rewritten as

$$
U(S \lambda, s, z, x)=(S \lambda)(x)-\frac{\beta}{2 s}-s R(x)+Y
$$

Agents are free to move across the space. Since they are identical, they must have the same utility level $u \in \mathbb{R}$. Landlords raise land rents until no agent has incentives to move. A resident with utility $u$ can propose to landlords the land bid $\Psi: \mathbb{R} \times \mathcal{F} \times \mathbb{R} \times \mathbb{R} \rightarrow \mathcal{F}$

$$
\Psi(s, S \lambda, u, x)=\frac{(S \lambda)(x)-\beta /(2 s)+Y-u}{s}
$$

Given land competition, each resident must offer her highest bid rent $\widehat{\Psi}: \mathcal{F} \times \mathbb{R} \times \mathbb{R} \rightarrow \mathcal{F}$, which corresponds to the maximum rent that she is willing to pay for dwelling in a given location: $\widehat{\Psi}: \max _{s} \Psi(s, S \lambda, u, x)$. Her optimal use of space $\widehat{s}: \mathcal{F} \times \mathbb{R} \times \mathbb{R} \rightarrow \mathcal{F}$ is given by

$$
\widehat{s}(S \lambda, u, x)=\arg \max _{s} \Psi(s, S \lambda, u, x)=\frac{\beta}{(S \lambda)(x)+Y-u}
$$

which gives

$$
\widehat{\Psi}(S \lambda, u, x)=\frac{\beta}{2}[\widehat{s}(S \lambda, u, x)]^{2}
$$

In locations where residents dwell, land market clears if $\lambda \widehat{s}(S \lambda, u, x)=1$.

Definition 1 A spatial equilibrium is a utility level $u \in \mathbb{R}$, density and land rent functions $\lambda, R \in \mathcal{F}$ such that 
(i) landlords rent land to residents: $\lambda(x)=1 / \widehat{s}(S \lambda, u, x)>0$ and $R(x)=\widehat{\Psi}(S, u, x)$ if and only if $\widehat{\Psi}(S \lambda, u, x) \geq R_{A}$

(ii) landlords rent land to farming: $\lambda(x)=0$ and $R(x)=R_{A}$ if and only if $\widehat{\Psi}(S \lambda, u, x)<$ $R_{A}$;

(iii) all individuals find residence: $\int_{\mathbb{R}} \lambda \mathrm{d} x=M$.

Using (4), conditions (i) and (ii) can be restated as it follows:

(i) $\lambda(x)=\frac{1}{\widehat{s}(S \lambda, u, x)}$ and $R(x)=\frac{\beta}{2}[\widehat{s}(S \lambda, u, x)]^{2} \Longleftrightarrow \widehat{s}(S \lambda, u, x) \geq \sqrt{\frac{2 R_{A}}{\beta}}$

(ii) $\lambda(x)=0$ and $R(x)=R_{A} \Longleftrightarrow \widehat{s}(S \lambda, u, x)<\sqrt{\frac{2 R_{A}}{\beta}}$

where

$$
\lambda_{A} \equiv \sqrt{2 R_{A} / \beta}
$$

Using (3) and dropping $R(x)$, the above definition yields the following fixed point definition:

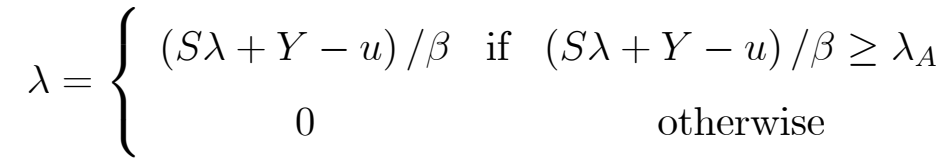

$$
\begin{aligned}
& M=\int_{\mathbb{R}} \lambda \mathrm{d} x
\end{aligned}
$$

Note that, if $\lambda_{A}>0$, population density $\lambda$ is larger than zero and the city with a mass $M$ of residents must have finite boundaries. As a consequence, $\lambda$ has jump(s) from above $\lambda_{A}$ to 0 about those boundaries.

\section{General properties}

In this section we outline some properties of the spatial equilibria for any travel cost function. For simplicity, we consider a city with compact support $[-b, b]$ so that $\lambda(x) \geq \lambda_{A}$ if $x \in[-b, b]$ and $\lambda(x)=0$ otherwise. 
First, we show that the equilibrium population density is smooth and has no abrupt changes on the city support. More precisely, the function $\lambda$ is continuous, once differentiable on $(-b, b)$ and infinitely differentiable on the set $(-b, b)$ minus a finite set of points. Indeed, we can make the interaction window explicit in the integral boundaries and write (5) as

$$
\lambda(x)=\frac{1}{\beta} \int_{x-a}^{x+a} A(x-y) \lambda(y) \mathrm{d} y+\frac{1}{\beta}(Y-u)
$$

if $x \in(-b, b)$ while $\lambda(x)=0$ if $x \in(-\infty,-b) \cup(b, \infty)$. The density function $\lambda$ inherits the continuity property of the above integral and is therefore continuous and differentiable on $(-b, b)$. Therefore, given that $\lambda$ is zero for $x \in(-\infty,-b) \cup(b, \infty), \lambda$ is continuous and differentiable on $\mathbb{R} \backslash\{-b, b\}$. Similarly, since $A$ is continuous and $\lambda$ is continuous and differentiable on $\mathbb{R} \backslash\{-b, b\}, \lambda^{\prime}$ is continuous and differentiable on $\mathbb{R} \backslash\{-b+a, b-a\}$. Repeating the argument, one can see that $\lambda^{(i)}$ is continuous and differentiable on $(-b, b)$ except on the set of points in $\pm(b-i a), i \in \mathbb{N}_{0}$ where $\mathbb{N}_{0}$ is the set of non-zero natural numbers.

Second, we show that the equilibrium population density is symmetric about a central location. Let us denote the central location as $x=0$ w.l.o.g. We can apply (6) at $-x$ and make the change of variable $y=-z$ in (6) we get

$$
\lambda(-x)=\frac{1}{\beta} \int_{x-a}^{x+a} A(-x+z) \lambda(-z) d z+\frac{1}{\beta}(Y-u)
$$

Given the symmetry of $A, \lambda(-x)=\lambda(x)$. Furthermore, the boundary conditions $\lambda(b)=$ $\lambda(-b)=\lambda_{A}$ and density condition $\int_{-b}^{b} \lambda(y) d y=M$ are also satisfied when we replace $\lambda(x)$ by $\lambda(-x)$.

Third, the structure of the spatial equilibrium is invariant to some parameter changes. Let us define the economy $\mathcal{E}\left(b, \lambda ; \tau, a, \beta, R_{A}, M\right)$ where the two first elements are the equilibrium city border and density and the five other elements are the exogenous parameters of the model. We then have the following correspondences between equilibria:

Proposition $2 \mathcal{E}\left(b, \lambda ; \tau, a, \beta, R_{A}, M\right)$ is equivalent to 
1. $\mathcal{E}\left(b, \lambda / \sqrt{R_{A}} ; \tau, a, \beta, 1, M / \sqrt{R_{A}}\right)$,

2. $\mathcal{E}\left(b, \lambda / M ; \tau, a, \beta, R_{A} / M^{2}, 1\right)$,

3. $\mathcal{E}\left(\tau b, \lambda(x / \tau) ; 1, \tau a, \tau \beta, \tau R_{A}, \tau M\right)$

Proof. See Appendix A.

This means that an increase in the opportunity cost of land $R_{A}$ leads to the same spatial equilibrium with proportionally smaller population density and mass but no change in the city border. Similarly after a rise in its total population, a city keeps the same spatial structure with a proportionally smaller population density, provided that the opportunity cost of land falls more than proportionally $\left(1 / M^{2}\right)$. Finally, a fall in the travel cost parameter $\tau$ sustains the same spatial structure with proportional "horizontal" expansion provided that the interaction window, the preference for land, the opportunity cost of land and the population grow in the same proportion.

The proposition also implies that two parameters out of the five are redundant. We can construct the spatial equilibrium from one obtained with another set of parameters. In the case of linear travel cost, three parameters are redundant since then $a=\tau^{-1}$.

Finally, since population densities are continuous functions and the equilibrium is defined by the fixed point of an operator that maps the space of such continuous density function on itself, the Contraction Mapping theorem can be used to show existence and uniqueness of a spatial equilibrium. The main difficulty in this approach lies in the presence of a opportunity cost of land $R_{A}>0$, which implies to guarantee a minimal density level $\lambda_{A}$ within the city support and a zero density outside it.

Proposition 3 Consider any city support $[-b, b]$. Then, if $b<\beta / 2$, there exists a unique spatial equilibrium with positive population size $M>0$.

Proof. See Appendix B. 


\section{Linear travel cost}

We here consider the case of linear travel cost $T(\tau x)=\tau|x|$ so that $a=1 / \tau$. This case will allow us to discuss the impact of local interaction and to discuss spatial equilibrium solution. Note that the travel cost has a kink at $x=0$.

Agents reach the same utility over all inhabited locations. This gives the basic spatial equilibrium relationship as it follows. For convenience, define the operator $V: \mathcal{F} \rightarrow \mathcal{F}$ that returns the spatial distribution of utility within a city,

$$
(V \lambda)(x) \equiv(S \lambda)(x)-\beta \lambda(x)+Y
$$

Then, by (5), a spatial equilibrium imposes $(V \lambda)(x)=u$ for all $x$ such that $((S \lambda)(x)+Y-u) / \beta \geq$ $\lambda_{A}$. Because $u$ is a constant, it must be that $\left(\mathrm{d}^{m} / \mathrm{d}^{m} x\right)(V \lambda)=0, m=1,2, \ldots$ Because

$$
\frac{\mathrm{d}}{\mathrm{d} x}(S \lambda)=\tau \int_{x}^{x+a} \lambda(y) \mathrm{d} y-\tau \int_{x-a}^{x} \lambda(y) \mathrm{d} y
$$

a first necessary equilibrium condition is $(\mathrm{d} / \mathrm{d} x)(V \lambda)=0$. That is,

$$
\lambda^{\prime}(x)=\tau \beta^{-1} \int_{x}^{x+a} \lambda(y) \mathrm{d} y-\tau \beta^{-1} \int_{x-a}^{x} \lambda(y) \mathrm{d} y
$$

One can differentiate further $S \lambda$ and get

$$
\frac{\mathrm{d}^{2}}{\mathrm{~d} x^{2}}(S \lambda)=\tau[-2 \lambda(x)+\lambda(x+a)+\lambda(x-a)]
$$

Another necessary equilibrium condition is $\left(\mathrm{d}^{2} / \mathrm{d} x^{2}\right)(V \lambda)=0$, which leads to

$$
\lambda^{\prime \prime}(x)=-\delta^{2}\left[\lambda(x)-\frac{1}{2} \lambda(x+a)-\frac{1}{2} \lambda(x-a)\right]
$$

where

$$
\delta^{2} \equiv 2 \tau / \beta
$$

This is Mixed-type Functional Differential Equation (MFDE), which, to our knowledge, explicit solutions have not been reported in the literature. The advance and delay are caused by the fact that interaction benefit vanishes outside each agent's interaction window. 


\subsection{Global and local interactions}

To our knowledge, the literature on urban and regional interactions is rooted on the assumption of global interactions. Under global interactions, agents are able to benefit from meeting every other agent at any distance within the considered area. By contrast, under local interactions, agents do not benefit from meeting other agents residing at too distant locations within the considered area. We here explain the implications of such a spatial window of interactions.

First, consider a single city in an interval $[-b, b]$. Because spatial equilibria are symmetric, $\lambda$ is symmetric about $x=0$. Global interactions take place when $\tau$ is small enough so that any location $x \pm a$ lies outside the city where no agent lives: $\lambda(x+a)=\lambda(x-a)=0$. The differential equation (9) simplifies to

$$
\lambda^{\prime \prime}(x)=-\delta^{2} \lambda(x)
$$

with no advances and delays. Therefore, the population density $\lambda$ has the mathematical form of $\cos (\delta x)$ and its amplitude is determined by the population constraint $\int_{-b}^{b} \lambda(x) \mathrm{d} x=$ $M$. One readily checks that the equilibrium population density is given by

$$
\lambda(x)=\frac{\delta M}{2 \sin (\delta b)} \cos (\delta x), \quad x \in[-b, b]
$$

As a result, $\lambda$ is bell shaped, and so is $R=\beta \lambda^{2} / 2$. The border land arbitrage condition $R( \pm b)=R_{A}$ implies $\lambda( \pm b)=\lambda_{A}$ and gives the equilibrium city border $b=$ $\frac{1}{\delta} \arctan \left(\frac{1}{2} \delta M / \lambda_{A}\right)$. Ceteris paribus, the city border increases for larger population size $M$ and lower land rent $R_{A}$. The relationship between border and population mass can also be inverted as

$$
\mathcal{M}(b)=2 \frac{\lambda_{A}}{\delta} \tan \delta b=\lambda_{A} \sqrt{\frac{2 \beta}{\tau}} \tan \left(\sqrt{\frac{2 \tau}{\beta}} b\right)
$$

This describes the spatial structure of a single city for large enough interaction window a. 


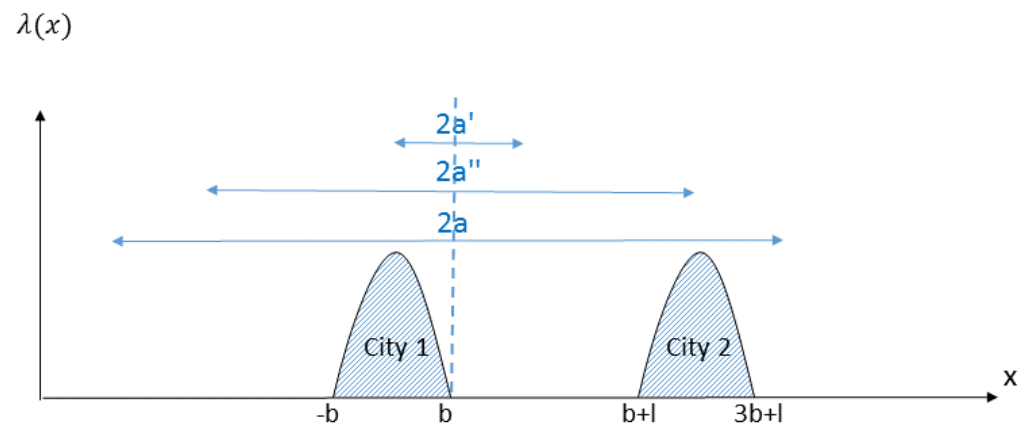

Figure 1: Density, interaction window and distance between cities

Under the assumption of global interactions, agents should be able to meet every other. That is, the city should not expand too much: formally,

$$
2 b<a
$$

should hold.

The assumption of global interaction restrains the number of cities emerging at the equilibrium on an open line. Figure 1 shows this case of global interaction when the window size $2 a$ covers the two cities: $a>2 b+l$. Picard and Mossay (2011) show that, if two cities existed as depicted in Figure 1, agents located at the outer border of each city $(x=-b$ and $x=3 b+l)$ have incentives to relocate at the inner border of their own city. Doing that, they do not get farther from the agents in their own city but get closer to the agents in the other city. This case implies global interactions but is not a spatial equilibrium. 
By contrast, when interactions are local, the interaction window is limited in distance. As shown in Figure 1 for the window size $2 a^{\prime}$ with $a^{\prime}<l$, a configuration with two cities is a spatial equilibrium provided that the hinterland length $l$ between the cities is larger than the interaction window half size $a$. Then, no agent in a city has an incentive to interact with an agent in a neighboring city because the latter is too far and gives no net benefit. As a consequence, a spatial equilibrium may include any number of cities with identical internal urban structure.

The impact of local interaction is less clear when interaction windows include the support of a city but only a share of the support of another city as shown in Figure 1 for the interaction window size $2 a^{\prime \prime}$ with $a^{\prime \prime} \in[l, 2 b+l)$. In this case, agents in a city get an interaction benefit only with a share of their partners in the other city and it is not sure whether agents have incentives to relocate. It turns out that a spatial equilibrium cannot sustain such two cities. It is shown in the proof of the following Proposition that agents about city borders $b$ or $b+l$ have incentives to relocate within the empty hinterland.

Proposition 4 Under local interactions and linear travel, there exists no spatial equilibrium with two cities separated by a hinterland length $l$ with $l<a$. Spatial equilibria may however include any number of symmetric cities separated by larger hinterlands.

\section{Proof. See Appendix C.}

Intuitively, in any urban area, there can be no "gap" smaller than the half of the interaction window.

In this section we have clarified the concepts and implications of the assumptions of global and local interactions. The assumption of global interactions eliminates the possibility of multiple cities. The objective of the following section is to understand the internal city structure under local interaction, i.e. when (11) does not hold. 


\subsection{Constructing spatial equilibria}

In the initial problem the city border $b$ is endogenous. However it is simpler to characterize the spatial equilibrium on a given city support $[-b, b]$ and then determine the urban population mass $\mathcal{M}(b)=\int_{-b}^{b} \lambda(x) \mathrm{d} x$. So, we consider $b$ as exogenous in this section.

We have the following necessary conditions:

$$
\begin{aligned}
& \ddot{\lambda}(x)=-\delta^{2}\left[\lambda(x)-\frac{1}{2} \lambda(x+a)-\frac{1}{2} \lambda(x-a)\right], x \in(-b, b) \\
& \lambda(x)=0, x \in(-b-a, b) \cup(b, b+a) \\
& \lambda(b)=\lambda(-b)=\lambda_{A}
\end{aligned}
$$

where $\delta^{2}=2 \tau / \beta$ and $\lambda_{A}=\sqrt{2 R_{A} / \beta}$. Those conditions are sufficient if $V^{\prime}(0)=0$ and $\lambda(x) \geq \lambda_{A}$ for $x \in[-b, b]$. However, because an equilibrium population density is symmetric about the central location $x=0$, we readily get that $V^{\prime}(0)=0$. Since $(12)$ is equivalent to $V^{\prime \prime}(x)=0, x \in(-b, b)$, the latter condition implies that $V^{\prime}(x)=0$, $x \in(-b, b)$. The second sufficient condition $\lambda(x) \geq \lambda_{A}$ for $x \in[-b, b]$ will be checked later.

The main mathematical innovation is to provide the basis of the solutions of the second order MFDE (12). Whereas the solution basis of the ordinary second order differential equation is found in the class of exponential and trigonometric functions on the support of the city $[-b, b]$, the solution basis of (12) must be found on a set of smaller supports on which the population density $\lambda$ is infinitely differentiable. As mentioned above, those are the intervals lying between the set of points $\pm(b-i a), i \in \mathbb{N}_{0}$.

Intuitively, any agent located at $x$ have an interaction window $[x-a, x+a]$. The agent located at $x \in(-b,-b+a)$ is not able to fully exploit her interaction window because there are no agents in the interval $[x-a,-b)$ on her left hand side. By contrast, an agent located at $x \in(-b+a,-b+2 a)$ is able to interact with agents on her left and right hand interaction windows. The agent located at $x=-b+a$ is therefore critical in term of the use of the interaction window. In equilibrium, agents on the left side of this point should be compensated for their fewer interaction by larger residential space so that the 
population density should be lower there too. In the same way, agents located in the next interval $(-b+a,-b+2 a)$ interact at their left and right hand side. But, since the population density is lower to the right of $x=-b+a$, they obtain a lower net benefit from their right hand side compared to their left hand side. The agent located at $x=-b+2 a$ is also critical. It can easily be understood that agent choices will depend on the set of contiguous windows of length $a$ starting from the left hand side. By the same argument, the agents' choices should also depend on the set of contiguous windows with the same length starting from the other side. The intersection of those windows gives the partition of the urban space on which we find for a basis of solutions for (12), (13) and (14). This decomposition is also proposed in Guinn (1976), Mallet-Paret and Verduyn-Lunel (2001).

Let $n \in \mathbb{N}_{0}$ be the maximum number of contiguous windows of length $a$ embedded the city support $[-b, b]$. It satisfies the inequalities $a n \leq 2 b<a(n+1)$. Let also $c=2 b-n a \in$ $[0, a)$ be the remaining space after placement of those $n$ windows. We then partition the city support into $2 n+1$ contiguous intervals with alternating lengths $c$ and $a-c$. That is, the interval $[-b, b]$ is partitioned in a sequence of interval of lengths: $c, a-c, c \ldots, c, a-c, c$. There are $n^{o}=n+1$ intervals with length $c$ and $n^{e}=n$ of intervals with length $a-c$. The total number of interval is denoted by $N=2 n+1$. For convenience, we define the density functions $\mu_{i}^{o}$ and $\mu_{i}^{e}$ that shift the support of the density $\lambda$ respectively onto the interval $[0, c]$ and $[0, a-c]$. That is,

$$
\begin{aligned}
& \mu_{i}^{o}(x):[0, c] \rightarrow \mathbb{R}^{+} \text {s.t. } \mu_{i}^{o}(x)=\lambda(x-b+(i-1) a), i \in\left\{1, \ldots, n^{0}\right\}, \\
& \mu_{i}^{e}(x):[0, a-c] \rightarrow \mathbb{R}^{+} \text {s.t. } \mu_{i}^{e}(x)=\lambda(x-b+c+(i-1) a), i \in\left\{1, \ldots, n^{e}\right\}
\end{aligned}
$$

Also, we define

$$
\begin{aligned}
& \mu_{i}^{o}(x):[0, c] \rightarrow \mathbb{R}^{+} \text {s.t. } \mu_{i}^{o}(x)=0, i \in\left\{0, n^{o}+1\right\} \\
& \mu_{i}^{e}(x):[0, a-c] \rightarrow \mathbb{R}^{+} \text {s.t. } \mu_{i}^{e}(x)=0, i \in\left\{0, n^{e}+1\right\}
\end{aligned}
$$

in order to take into account the null density outside the city.

Let us denote $l^{e}=c$ and $l^{o}=a-c$ for the sake of convenience. We denote the vectors of functions as the (column) vector $\mu^{s}=\left(\mu_{1}^{s}, \ldots, \mu_{n^{s}}^{s}\right)^{\prime}, s \in\{o, e\}$, where the apostrophe 
denotes the vector transpose. Then, for each $s \in\{o, e\}$, the delay differential equation (12) is equivalent to the following system of ordinary differential equations

$$
\begin{aligned}
& \ddot{\mu}_{i}^{s}(x)=-\delta^{2} \mu_{i}^{s}(x)+\frac{\delta^{2}}{2} \mu_{i+1}^{s}(x)+\frac{\delta^{2}}{2} \mu_{i-1}^{s}(x), \quad i \in\left\{1, \ldots, n^{s}\right\} \\
& \mu_{0}^{s}(x)=\mu_{n^{s}+1}^{s}(x)=0
\end{aligned}
$$

for all $x \in\left(0, l^{s}\right)$. Given this spatial decomposition, the population density on each interval is given by the following basis of functions:

Lemma 5 The vector of equilibrium (shifted) population densities are given by

$$
\mu^{s}(x)=\sum_{j=1}^{n^{s}} g_{j}^{s} v_{j}^{s} \cos \left(\omega_{j}^{s} x\right)+f_{j}^{s} v_{j}^{s} \sin \left(\omega_{j}^{s} x\right)
$$

where

$$
\begin{aligned}
& \omega_{j}^{s}=\delta \sqrt{1-\cos \left(\frac{\pi j}{n^{s}+1}\right)}, j=1 \ldots n^{s} \\
& v_{j}^{s}=\sqrt{\frac{2}{n^{s}+1}}\left(\sin \left(\frac{\pi j}{n^{s}+1}\right), \sin \left(\frac{2 \pi j}{n^{s}+1}\right) \ldots, \sin \left(\frac{n^{s} \pi j}{n^{s}+1}\right)\right)^{\prime}, j=1 \ldots n^{s}
\end{aligned}
$$

each pair $\left(g_{j}^{s}, f_{j}^{s}\right) \in \mathbb{R}^{2}$ are constants and $s \in\{o, e\}$.

Proof. The system (15) can be written as $\ddot{\mu}^{s}(x)=T_{o}^{s} \mu^{s}(x)$ where $T_{o}^{s}$ is a $n^{s} \times n^{s}$ Toeplizt matrix with diagonal elements $\left(T_{o}^{s}\right)_{i, i}$ equal to $-\delta^{2}$ and super- and sub-diagonal elements $\left(T_{o}^{s}\right)_{i, i+1}$ and $\left(T_{o}^{s}\right)_{i+1, i}$ equal to $\delta^{2} / 2$. For instance, for $n^{s}=3$, it is given by

$$
T_{o}^{s}=-\frac{\delta^{2}}{2}\left(\begin{array}{ccc}
2 & -1 & 0 \\
-1 & 2 & -1 \\
0 & -1 & 2
\end{array}\right)
$$

The eigenvalues $-\left(\omega_{k}^{s}\right)^{2}$ and eigenvectors are shown in the Lemma.

Border and pasting conditions determine the $2 N$ constants $g_{i}^{s}$ and $f_{i}^{s}$. The border conditions (14) are equivalent to

$$
\mu_{1}^{e}(0)=\mu_{n^{e}}^{e}\left(l^{e}\right)=\lambda_{A}
$$


while continuity and differentiability at the border between pieces impose

$$
\begin{aligned}
& \mu_{i}^{e}\left(l^{e}\right)=\mu_{i}^{o}(0) \quad \text { and } \quad \dot{\mu}_{i}^{e}\left(l^{e}\right)=\dot{\mu}_{i}^{o}(0), \quad i=1, \ldots, n^{e}-1 \\
& \mu_{i}^{o}\left(l^{o}\right)=\mu_{i+1}^{e}(0) \quad \text { and } \quad \dot{\mu}_{i}^{o}\left(l^{o}\right)=\dot{\mu}_{i+1}^{e}(0), \quad i=1, \ldots, n^{o}
\end{aligned}
$$

This is a system of $2 N$ linear equations with $2 N$ unknowns. The solution of this system is shown in Appendix D.

Stacking the constants in the $2 N$ (column) vector $h=\left(g_{1}^{e}, \ldots, g_{n^{e}}^{e}, f_{1}^{e}, \ldots, f_{n^{e}}^{e}, g_{1}^{o}, \ldots, g_{n^{o}}^{o}\right.$, $\left.f_{1}^{o}, \ldots, f_{n^{o}}^{o}\right)^{\prime}$, the above conditions write in the matrix form

$$
Q h=\lambda_{A} q
$$

where $q=(-1,0, \ldots, 0,1,0, \ldots, 0)^{\prime}$ is a $2 N$ (column) vector and $Q$ is a $2 N \times 2 N$ matrix given in Appendix D. The properties of the matrix $Q$ and vector $\lambda_{A} q$ give information on the existence and number of solution vectors $h$. Since those solution vectors correspond to spatial equilibria only if they yield a population density $\lambda$ that is everywhere larger than $\lambda_{A}$ on the city support, those properties give an upper bound on the number of spatial equilibria. Let

$$
r=\operatorname{rank}(Q)
$$

and remind that $\lambda_{A}=\sqrt{2 R_{A} / \beta}$. We therefore get the following lemma:

Lemma 6 If $\operatorname{det}(Q) \neq 0$, there exists at most one non-zero spatial equilibrium. Otherwise, the set of non-zero spatial equilibria has at most dimension $2 N-r$.

\section{Proof. See Appendix D.}

Note that, since matrix $Q$ is a linear combination of trigonometric functions of economic parameters, it accepts a zero determinant only for a zero-measure set of parameters. The case where $\operatorname{det}(Q)=0$ is therefore not generic. We therefore focus on the case of a unique solution when $\operatorname{det} Q \neq 0$.

The vector of constants $h$ are determined by inverting this system as $h=\lambda_{A} Q^{-1} q$. From this we can recover the vector $g^{s}$ and $f^{s}$, which are proportional to $\lambda_{A}$ and therefore $\sqrt{R_{A}}$. The city population mass is given by $\mathcal{M}(b)=\sum_{i=1}^{n^{o}} m_{i}^{o}+\sum_{i=1}^{n^{e}} m_{i}^{e}$ where $m_{i}^{s}=$ 
$\int_{0}^{l^{s}} \mu_{i}^{s}(x) \mathrm{d} x=\sum_{j=1}^{n^{s}} \frac{v_{i j}^{s}}{\omega_{j}^{s}}\left\{g_{j}^{s} \sin \left(\omega_{j}^{s} l^{s}\right)-f_{j}^{s}\left[1-\cos \left(\omega_{j}^{s} l^{s}\right)\right]\right\}$ are the population masses in each piece $i \in\left\{1, \ldots, n^{o}+n^{e}\right\}$. We therefore have proven the following: each combination of parameters $\left(a, \tau, \beta, R_{A}\right)$ and city border $b$ yields at most one non-zero spatial equilibrium with finite population mass $M$ on the support $[-b, b]$. This does not guarantee that population densities are high enough. However, by Proposition 3, we know that the spatial equilibrium with $\lambda(x)>\lambda_{A}$ exists and is unique for $b<\beta / 2$.

Proposition 7 Suppose $\operatorname{det}(Q) \neq 0$. Then, each combination of parameters $\left(a, \tau, \beta, R_{A}\right)$ and city border $b<\beta / 2$ yields a unique non-zero spatial equilibrium with finite population mass $M$. The population mass is proportional to $\sqrt{R_{A}}$.

That is, a city border $b<\beta / 2$ maps to a population mass for a generic set of economic parameters. For $b>\beta / 2$, the solutions of (20) may yield a population density lower than $\lambda_{A}$ within the city support so that a spatial equilibrium may fail to exist. Also, there may be two city borders $b$ and $b^{\prime}$ that yield spatial equilibria with same population mass $M=M^{\prime}$. To our knowledge, only numerical analyses can put light on those two issues.

\subsection{Numerical analysis}

We can here study the properties of the city population distributions and masses. By Proposition 2, we can normalize $\lambda_{A}$ and $\tau$ to 1 so that $a=1$. We just need to study the spatial equilibria for various $\beta$ and $b$. For $a>2 b$, all agents in the city have global interaction so that the relationship between border and population mass (10) becomes $\mathcal{M}(b)=\tan (2 b)$, which the population mass varies between 0 and $\tan a=1.557$.

Figure 2 shows the equilibrium population $\mathcal{M}(b)$ as a function of the city border $b$ when cities are subject to local interaction: $a<2 b$. The solid curve shows the constructed equilibrium populations that satisfy the condition $\lambda(z) \geq \lambda_{A}, z \in[-b, b]$. The dashed curves display the populations in constructed cities that are not equilibria; that is, in which we numerically spot a location $z \in[-b, b]$ with $\lambda(z)<\lambda_{A}$. The apparent property is that, 
there exists a unique spatial equilibrium with a finite border $b>a / 2$ for any population mass $M$ larger than $\tan a$. So, merging the cases of global and local interactions, there exists a unique spatial equilibrium with a finite border for any population mass.

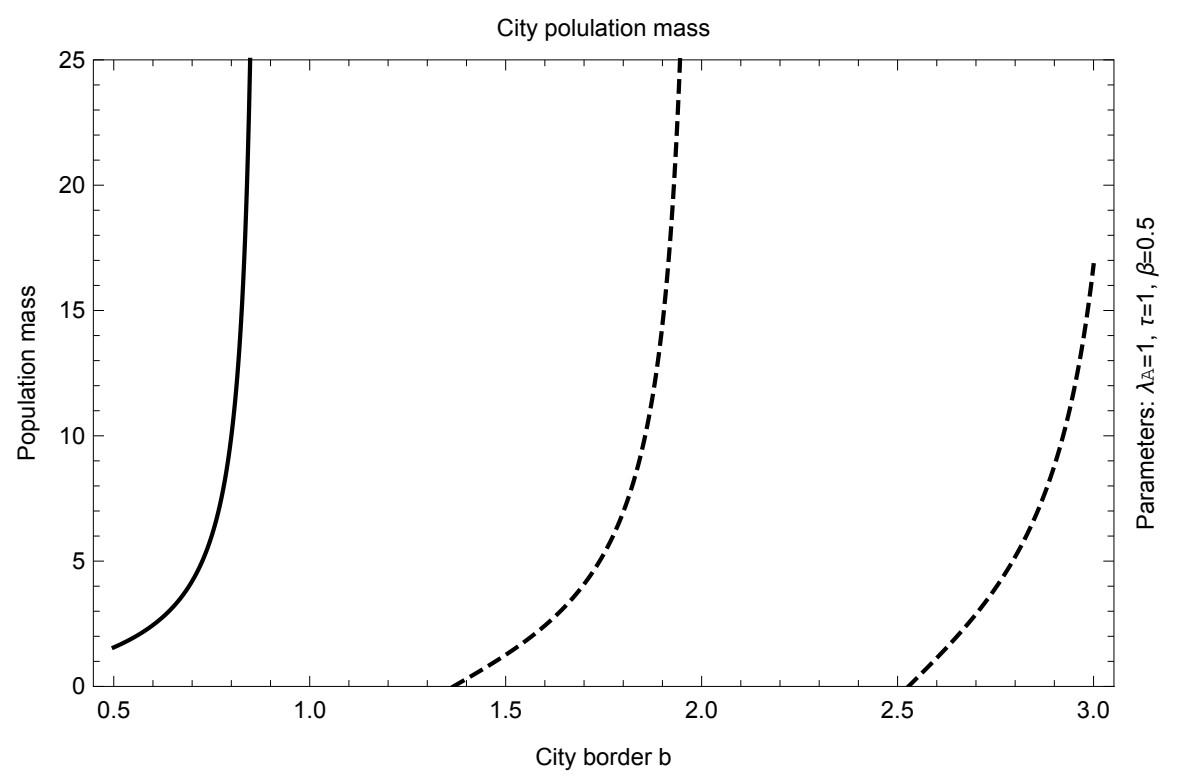

Figure 2: Population masses as function of city border (solid line=equilibrium; dashed $=$ not equilibrium).

It is also noticeable that, whatever the population mass, the city extends to a maximum length (that is lower than 1 in Figure 2). In such situation, agents residing at the city center find a net benefit to interact with all other agents whereas those residing at the city edge refrain from interacting with too distant agents. So, local interaction limits the extent of the city. This maximum city length depends on land preferences. Figure 3 shows the same relationships for various parameters for residential land preference $\beta \in$ $\{0.25,0.50,0.75\}$. A stronger preference for residential land increases the maximum city extent. 


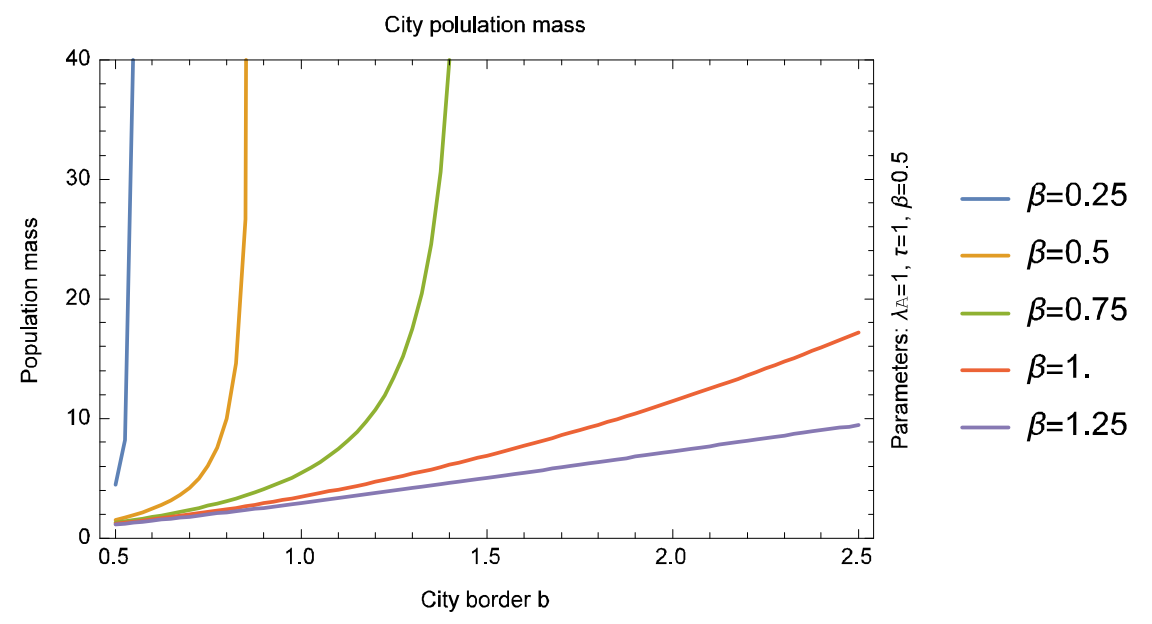

Figure 3: Population masses for various land preference parameters.

Can there be polycentric cities? Figure 4 displays the population density of cities with the same population size for various interaction windows. The smaller the interaction window, the flatter the population density and the larger the city extent. The figure shows the emergence of a unique centers where population density peaks. Using other parameters, we have found no numerical instances with more than one such center.

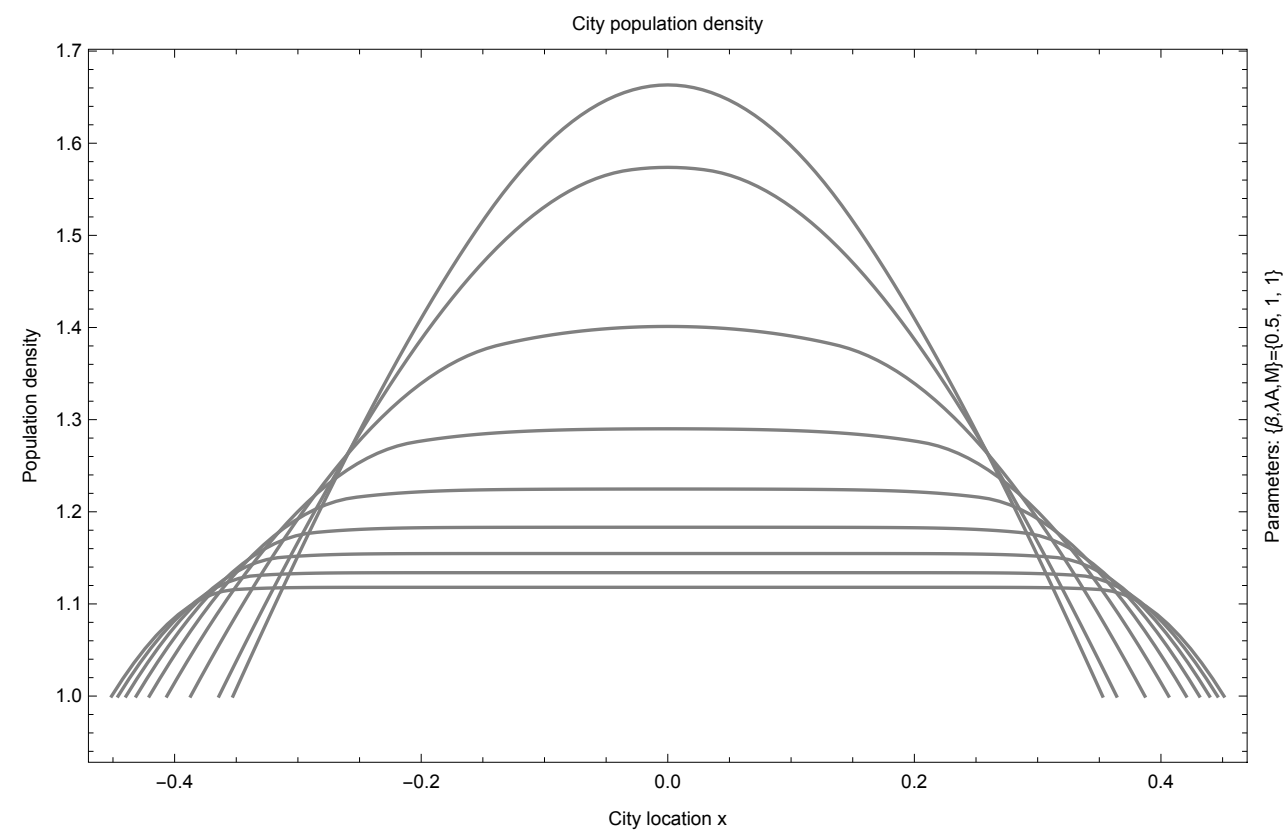

Figure 4: Population density and interaction window sizes. 


\section{Conclusion}

In this paper we investigate the effect of local interaction in a simple urban economics model. Agents interact with others if only if they get a benefit net of their travel cost. As a consequence, each agent entertain interaction within a finite interaction window. We show that two or more cites may coexist at the equilibrium provided that they are sufficiently distant. The population density within a city determined by a second order Mixed-type Functional Differential Equation, which solves as a piecewise trigonometric function. Supposing that urban residents are able to outbid the land value for non-urban use (e.g. farming), we characterize equilibrium candidates as piecewise trigonometric function of residents' coordinates. Apply the Contraction Mapping Theorem, we infer that each candidate is a unique spatial equilibrium in cities with not too large geographical extents. Numerical simulations show that residents are indeed able to outbid external users. Our study confirms that usual properties of urban equilibria apply under local interactions. In addition, we show that more localized interactions tend to yield flatter population density and land rents over a larger extent of the city support. We do not find support to the idea that local interaction may generate cities with multiple subcenters.

Overall, our study highlights an unknown and interesting application of advanceddelay differential equation in urban economics. Our results may be generalized for non linear interaction surplus functions. In those cases, candidate equilibria become piecewise functions of agents' coordinates, that are defined on the same partitions of city supports and are solutions of non-linear MFDF differential equations. All in all, the assumption of local interaction necessitates mathematical tools going beyond the ones used in standard urban economics. Whereas a complete analytical characterization of spatial equilibria proves difficult, basic equilibrium properties however remain standard. 


\section{References}

d'Albis, H., Augeraud-Véron, E. (2007). Balanced cycles in an OLG model with a continuum of finitely-lived individuals. Economic Theory, 30(1), 181-186.

d'Albis, H., Augeraud-Véron, E. and Hupkes, H. J. (2014). Stability and determinacy conditions for mixed-type functional differential equations. Journal of Mathematical Economics, 53, 119-129.

Beckmann, M.J. (1976), Spatial Equilibrium and the Dispersed City, in: Y.Y. Papageorgiou(ed.), Mathematical Land Use Theory, Lexington, MA:Lexington Books, 117-125.

Berliant, M., S.-K. Peng, and Wang, P. (2002) Production externalities and urban configuration. Journal of Economic Theory 104, 275-303.

Blanchet, A., Mossay, P. and Santambrogio, F. (2016). Existence and uniqueness of equilibrium for a spatial model of social interactions. International Economic Review, $57(1), 31-60$.

Büchel K. and von Ehrlich M. (2016). Cities and the Structure of Social Interactions: Evidence from Mobile Phone Data, Discussion paper 1608, University of Bern.

Boucekkine, R., Licandro, O., Puch, L. A., and Del Rio, F. (2005). Vintage capital and the dynamics of the AK model. Journal of economic theory, 120(1), 39-72.

Diekmann O., van Giles S., Verduyn Lunel S., Walter H.O. (1995). Delay Equations: Functional-, Complex-, and Nonlinear Analysis, Springer-Verlag, New York.

Frisch, R., \& Holme, H. (1935). The characteristic solutions of a mixed difference and differential equation occuring in economic dynamics. Econometrica: Journal of the Econometric Society, 225-239.

Fujita, M., \& Ogawa, H. (1982). Multiple equilibria and structural transition of nonmonocentric urban configurations. Regional science and urban economics, 12(2), 161-196. Guinn T. (1976). Reduction of delayed optimal control problems to nondelayed problems. Journal of Optimization Theory and Application, 18(3), 371-377. 
James, R. W., and Belz, M. H. (1938). The significance of the characteristic solutions of mixed difference and differential equations. Econometrica, Journal of the Econometric Society, 326-343.

Levy M. and Goldenberg J. (2014) The Gravitational Law of Social Interaction. Physica A 393, 418-426.

Lucas, R. E., \& Rossi-Hansberg, E. (2002). On the internal structure of cities. Econometrica, 70(4), 1445-1476.

Mallet-Paret J. and S. M. Verduyn-Lunel (2001). Exponential dichotomies and WienerHopf factorizations for mixed-type functional differential equations, forthcoming in J. of Differential Equations, to appear.

Mok D., B.Wellman, J.Carrasco (2010). Does distance matter in the age of the internet? Urban Studies 47, 2747-2.

Mossay P., \& Picard P. M. (2011). On spatial equilibria in a social interaction model. Journal of Economic Theory, 146(6), 2455-2477.

Mossay P. and Picard. P.M. (2019). Spatial segregation and urban structure. Forthcoming in Journal of Regional Science.

Mossay, P., Picard P.M. and Tabuchi T. (2017). Spatial Structures with Forward and Backward Linkages. CREA DP 2017-22.

\section{Appendix A: Invariances}

The spatial equilibrium solves

$$
\left\{\begin{array}{c}
\lambda(x)=\frac{1}{\beta} \int_{x-a}^{x+a} A(x-y) \lambda(y) d y+\frac{1}{\beta}(Y-u) \\
\lambda(b)=\lambda(-b)=\sqrt{\frac{2 R_{A}}{\beta}} \\
\int_{-b}^{b} \lambda(y) d y=M
\end{array}\right.
$$


For this problem, $\lambda, b$ and $u$ are endogenous. Because $u=\int_{b-a}^{b} A(b-y) \lambda(y) d y+Y-$ $\sqrt{2 \beta R_{A}}$, the first line of the problem can be written as

$$
\lambda(x)=\frac{1}{\beta} \int_{x-a}^{x+a} A(x-y) \lambda(y) d y+\sqrt{\frac{2 R_{A}}{\beta}}-\frac{1}{\beta} \int_{b-a}^{b} A(b-y) \lambda(y) d y
$$

which eliminates both $u$ and $Y$. The economy is described by $\mathcal{E}\left(b, \lambda ; \tau, a, \beta, R_{A}, M\right)$.

We first prove that $\mathcal{E}\left(b, \lambda ; \tau, a, \beta, R_{A}, M\right)$ is equivalent to $\mathcal{E}\left(b, \lambda / \sqrt{R_{A}} ; \tau, a, \beta, 1, M / \sqrt{R_{A}}\right)$ if $R_{A}>0$. Defining $\mu(x)=\lambda(x) / \sqrt{R_{A}}$ we have $\mu(b)=\mu(-b)=\sqrt{2 / \beta}$. The total population satisfies $\int_{-b}^{b} \mu(y) d y=M / \sqrt{R_{A}}$ and

$$
\mu(x)=\frac{1}{\beta} \int_{x-a}^{x+a} A(x-y) \mu(y) d y+1-\frac{1}{\beta} \int_{b-a}^{b} A(b-y) \mu(y) d y
$$

The endogenous variable $b$ is not affected by this change of variable. The last expression is invariant to $R_{A}$, which proves the statement.

We then prove that $\mathcal{E}\left(b, \lambda ; \tau, a, \beta, R_{A}, M\right)$ is equivalent to $\mathcal{E}\left(b, \lambda / M ; \tau, a, \beta, R_{A} / M^{2}, 1\right)$. Let $\mu(x)=\lambda(x) / M$ so that $\int_{-b}^{b} \mu(y) d y=1$. Dividing the boundary condition by $M$ yields $\mu(b)=\mu(-b)=\frac{1}{M} \sqrt{2 R_{A} / \beta}$. Then we get

$$
\mu(x)=\frac{1}{\beta} \int_{x-a}^{x+a} A(x-y) \mu(y) d y+\sqrt{\frac{2 R_{A}}{\beta M^{2}}}-\frac{1}{\beta} \int_{b-a}^{b} A(b-y) \mu(y) d y
$$

which is invariant provided that $R_{A} / M^{2}$ remains constant.

We finally prove that $\mathcal{E}\left(b, \lambda ; \tau, a, \beta, R_{A}, M\right)$ is equivalent to $\mathcal{E}\left(\tau b, \lambda(x / \tau) ; 1, \tau a, \tau \beta, \tau R_{A}, \tau M\right)$.

We have

$$
\lambda(x)=\frac{1}{\beta} \int_{x-a}^{x+a}[1-T(\tau(x-y))] \lambda(y) d y+\sqrt{\frac{2 R_{A}}{\beta}}-\frac{1}{\beta} \int_{b-a}^{b}[1-T(\tau(b-y))] \lambda(y) d y
$$

Changing the variables $(x, y, a, b)$ by $(\widetilde{x} / \tau, \widetilde{y} / \tau, \widetilde{a} / \tau, \widetilde{b} / \tau)$ and defining $\mu(\widetilde{x}) \equiv \lambda\left(\frac{\widetilde{x}}{\tau}\right)$ we get

$$
\mu(\widetilde{x})=\frac{1}{\tau \beta} \int_{\widetilde{x}-\widetilde{a}}^{\widetilde{x}+\widetilde{a}}[1-T(\widetilde{x}-\widetilde{y})] \mu(\widetilde{y}) d \widetilde{y}+\sqrt{\frac{2 \tau R_{A}}{\tau \beta}}-\frac{1}{\tau \beta} \int_{\widetilde{b}-\widetilde{a}}^{\widetilde{b}}[1-T(\widetilde{b}-\widetilde{y})] \mu(\widetilde{y}) d \widetilde{y}
$$

The support of $\mu$ is $(-\widetilde{b}, \widetilde{b})=(-\tau b, \tau b)$. The solution $\mu$ is equal to $\lambda$ provided that $\tau a, \tau b$, $\tau \beta$ and $\tau R_{A}$ remains the same. The population mass is $M=\int_{-b}^{b} \lambda(y) d y=\frac{1}{\tau} \int_{-\widetilde{b}}^{\widetilde{b}} \mu(\widetilde{y}) d \widetilde{y}$. So, $\tau M$ should remain the same too. 


\section{Appendix B: Fixed Point and Contraction}

We here prove the existence of a fixed point of $(5)$ on a city support $[-b, b]$. Let $b>0$, $\lambda_{B}>\lambda_{A}>0, X=C\left([-b, b],\left[\lambda_{A}, \lambda_{B}\right]\right)$. In $X$ we consider the sup norm, denoted by $\|\cdot\|$. We redefine the fixed point as

$$
\lambda=(S \lambda+Y-u) / \beta \quad \text { if } \quad(S \lambda+Y-u) / \beta \geq \lambda_{A} \quad \forall x \in[-b, b]
$$

The mass of residents $M=\int_{-b}^{b} \lambda \mathrm{d} x$ is endogenously determined. Note that at the city border we have

$$
\lambda(b)=((S \lambda)(b)+Y-u) / \beta=\lambda_{A}
$$

Indeed, if $\lambda(b)>\lambda_{A}$, by continuity of $(S \lambda)(x)$, there exists a small $\varepsilon>0$ such that $((S \lambda)(b+\varepsilon)+Y-u) / \beta>\lambda_{A}$, which contradicts that $b$ is the city border. We thus have $Y-u=\beta \lambda_{A}-(S \lambda)(b)$ and eliminate $u$ in (21) so that

$\lambda(x)=\lambda_{A}+\frac{1}{\beta}[(S \lambda)(x)-(S \lambda)(b)] \quad$ if $\quad \lambda_{A}+\frac{1}{\beta}[(S \lambda)(x)-(S \lambda)(b)] \geq \lambda_{A} \quad \forall x \in[-b, b]$

Let us define a first operator $\mathcal{L}_{1}: X \rightarrow C([-b, b], \mathbb{R})$ by

$$
\begin{aligned}
\left(\mathcal{L}_{1} \lambda\right)(x) & =\lambda_{A}+\frac{1}{\beta}[(S \lambda)(x)-(S \lambda)(b)] \\
& =\lambda_{A}+\frac{1}{\beta} \int_{-b}^{b}(A(x-y)-A(b-y)) \lambda(y) d y
\end{aligned}
$$

Define a second operator $\mathcal{L}_{2}: X \rightarrow C\left([-b, b],\left[\lambda_{A}, \infty\right)\right)$ by

$$
\left(\mathcal{L}_{2} \lambda\right)(x)=\max \left\{\lambda_{A},\left(\mathcal{L}_{1} \lambda\right)(x)\right\}
$$

Then, the fixed point (22) is equivalent to the fixed point $\lambda=\mathcal{L}_{2} \lambda$. We now show that the fixed point accepts a solution because it includes continous and bounded operators. We further derive a condition for a contraction, under which the solution is unique.

Lemma $8 \mathcal{L}_{1}$ and $\mathcal{L}_{2}$ are continuous operators such that $\left\|\mathcal{L}_{1} \lambda-\mathcal{L}_{1} \mu\right\|$ and $\left\|\mathcal{L}_{2} \lambda-\mathcal{L}_{2} \mu\right\|$ are bounded by $2 b \beta^{-1}\|\lambda-\mu\|$ for any $\lambda, \mu \in X$. 
Proof. We first prove that $\mathcal{L}_{1}$ is a continuous and contracting mapping. For any $\delta>0$ there exists an $\varepsilon>0$ such that $\|\lambda-\mu\|<\varepsilon \Rightarrow\left\|\mathcal{L}_{1} \lambda-\mathcal{L}_{1} \mu\right\|<\delta$. Indeed, since $0 \leq A(z) \leq 1$ we have that

$$
\mid A(x-y)-A(b-y) \leq 1
$$

Thererore

$$
\begin{aligned}
\left\|\mathcal{L}_{1} \lambda-\mathcal{L}_{1} \mu\right\| & =\beta^{-1} \sup _{x \in[-b, b]}\left|\int_{-b}^{b}(A(x-y)-A(b-y))[\lambda(y)-\mu(y)] \mathrm{d} y\right| \\
& \leq \beta^{-1} \sup _{x \in[-b, b]} \int_{-b}^{b}|A(x-y)-A(b-y)||\lambda(y)-\mu(y)| \mathrm{d} y \\
& \leq \beta^{-1} \sup _{x \in[-b, b]} \int_{-b}^{b}|\lambda(y)-\mu(y)| \mathrm{d} y \\
& =2 b \beta^{-1}\|\lambda-\mu\|
\end{aligned}
$$

So, we just need to set $\varepsilon=(\delta \beta) /(2 b)$. The operator $\mathcal{L}_{1}$ is a contraction for $2 b \beta^{-1}<1$.

We then prove that $\mathcal{L}_{2}$ is also a continuous and contracting mapping. That is

$$
\left\|\mathcal{L}_{2} \lambda-\mathcal{L}_{2} \mu\right\|=\sup _{x \in[-b, b]}\left|\max \left\{\left(\mathcal{L}_{1} \lambda\right)(x), \lambda_{A}\right\}-\max \left\{\left(\mathcal{L}_{1} \mu\right)(x), \lambda_{A}\right\}\right|
$$

is bounded by a function of $\left\|\mathcal{L}_{1} \lambda-\mathcal{L}_{1} \mu\right\|$. Consider the sets

$$
\begin{aligned}
& \mathcal{A}_{1}=\left\{x \in[-b, b]:\left(\mathcal{L}_{1} \lambda\right)(x) \leq \lambda_{A} \leq\left(\mathcal{L}_{1} \mu\right)(x)\right\} \\
& \mathcal{A}_{2}=\left\{x \in[-b, b]:\left(\mathcal{L}_{1} \mu\right)(x) \leq \lambda_{A} \leq\left(\mathcal{L}_{1} \lambda\right)(x)\right\} \\
& \mathcal{A}_{3}=\left\{x \in[-b, b]:\left(\mathcal{L}_{1} \mu\right)(x) \geq \lambda_{A},\left(\mathcal{L}_{1} \lambda\right)(x) \geq \lambda_{A}\right\} \\
& \mathcal{A}_{4}=\left\{x \in[-b, b]:\left(\mathcal{L}_{1} \mu\right)(x) \leq \lambda_{A},\left(\mathcal{L}_{1} \lambda\right)(x) \leq \lambda_{A}\right\}
\end{aligned}
$$

So, $\cup_{i=1}^{4} \mathcal{A}_{i}=[-b, b]$. Let $Z(x)=\left|\max \left\{\left(\mathcal{L}_{1} \lambda\right)(x), \lambda_{A}\right\}-\max \left\{\left(\mathcal{L}_{1} \mu\right)(x), \lambda_{A}\right\}\right|$. Then, it comes that:

If $x \in \mathcal{A}_{1}$, then $Z(x)=\left|\left(\mathcal{L}_{1} \mu\right)(x)-\lambda_{A}\right| \leq\left|\left(\mathcal{L}_{1} \mu\right)(x)-\left(\mathcal{L}_{1} \lambda\right)(x)\right|$.

If $x \in \mathcal{A}_{2}$, then $Z(x)=\left|\left(\mathcal{L}_{1} \lambda\right)(x)-\lambda_{A}\right| \leq\left|\left(\mathcal{L}_{1} \lambda\right)(x)-\left(\mathcal{L}_{1} \mu\right)(x)\right|$.

If $x \in \mathcal{A}_{3}$, then $Z(x)=\left|\mathcal{L}_{1} \lambda(x)-\mathcal{L}_{1} \mu(x)\right|$.

If $x \in \mathcal{A}_{4}$, then $Z(x)=0 \leq\left|\mathcal{L}_{1} \lambda(x)-\mathcal{L}_{1} \mu(x)\right|$. 
That is, $|Z(x)| \leq\left|\left(\mathcal{L}_{1} \lambda\right)(x)-\left(\mathcal{L}_{1} \mu\right)(x)\right|, x \in[-b, b]$. Using the proof for $\mathcal{L}_{1}$, this implies that $\left\|\mathcal{L}_{2} \lambda-\mathcal{L}_{2} \mu\right\| \leq\left\|\mathcal{L}_{1} \lambda-\mathcal{L}_{1} \mu\right\| \leq 2 b \beta^{-1}\|\lambda-\mu\|$. Finally, $\mathcal{L}_{2}$ is a contraction if $2 b \beta^{-1}<1$.

The above argument shows that the fixed point is a contraction and therefore unique if

$$
\frac{2 b}{\beta}<1
$$

\section{Appendix C: Hinterland between cities}

To see this, consider the city interval $[-b, b]$ and the empty hinterland $(b, b+l)$ as shown in Figure 1. Suppose $a^{\prime \prime} \in[l, 2 b+l)$. We show that some agents about the city borders $b$ and $b+l$ have incentives to relocate in the empty hinterland. In this section we take the spatial distribution as given so that we ease our notation by replacing $(V \lambda)(x)$ and $(S \lambda)(x)$ by $V(x)$ and $S(x)$.

At the spatial equilibrium the agents residing at $x=b$ and $b+l$ get equal utility $V(b)=S(b)-\beta \lambda_{A}+Y=u$ and $V(b+l)=S(b+l)-\beta \lambda_{A}+Y=u$. By contrast, an agent relocating at any $x \in(b, b+l)$ would get the utility $V(x)=\max _{s} S(x)-\beta /(2 s)-s R_{A}+Y$, which solves as $V(x)=S(x)-\sqrt{2 \beta R_{A}}+Y=S(x)-\beta \lambda_{A}+Y$. Hence, the utility differential

$$
\begin{gathered}
V(b)-V(x)=S(b)-S(x) \\
V(b+l)-V(x)=S(b+l)-S(x)
\end{gathered}
$$

where $S(x)=\int_{x-a^{\prime \prime}}^{b}(1-\tau|x-y|) \lambda(y) \mathrm{d} y+\int_{b+l}^{x+a^{\prime \prime}}(1-\tau|x-y|) \lambda(y) \mathrm{d} y$ for $x \in(b, b+l)$, which derivative is $(\mathrm{d} / \mathrm{d} x) S(x)=\tau\left(\int_{x-a^{\prime \prime}}^{b} \lambda(y) \mathrm{d} y-\int_{b+l}^{x+a^{\prime \prime}} \lambda(y) \mathrm{d} y\right)$. Let us consider a small $\varepsilon>0$. We have

$$
\begin{gathered}
V(b)-V(b+\varepsilon)=-\varepsilon \frac{\mathrm{d} S}{\mathrm{~d} x}(b)=-\varepsilon \tau\left(\int_{b-a^{\prime \prime}}^{b} \lambda(y) \mathrm{d} y-\int_{b+l}^{b+a^{\prime \prime}} \lambda(y) \mathrm{d} y\right) \\
V(b+l)-V(b+l-\varepsilon)=\varepsilon \frac{\mathrm{d} S}{\mathrm{~d} x}(b+l)=\varepsilon \tau\left(\int_{b+l-a^{\prime \prime}}^{b} \lambda(y) \mathrm{d} y-\int_{b+l}^{b+l+a^{\prime \prime}} \lambda(y) \mathrm{d} y\right)
\end{gathered}
$$


Summing the two lines gives

$$
\left(\begin{array}{c}
(V \lambda)(b)-(V \lambda)(b+\varepsilon) \\
+(V \lambda)(b+l)-(V \lambda)(b+l-\varepsilon)
\end{array}\right)=\varepsilon \tau\left(\begin{array}{c}
-\int_{b-a^{\prime \prime}}^{b} \lambda(y) \mathrm{d} y+\int_{b+l}^{b+a^{\prime \prime}} \lambda(y) \mathrm{d} y \\
+\int_{b+l-a^{\prime \prime}}^{b} \lambda(y) \mathrm{d} y-\int_{b+l}^{b+l+a^{\prime \prime}} \lambda(y) \mathrm{d} y
\end{array}\right)
$$

where the RHS is negative because $\int_{b-a^{\prime \prime}}^{b} \lambda(y) \mathrm{d} y>\int_{b+l-a^{\prime \prime}}^{b} \lambda(y) \mathrm{d} y$ and $\int_{b+l}^{b+a^{\prime \prime}} \lambda(y) \mathrm{d} y<$ $\int_{b+l}^{b+l+a^{\prime \prime}} \lambda(y) \mathrm{d} y$ given that $a^{\prime \prime} \in[l, 2 b+l)$. So it must be that either $V(b)-V(b+\varepsilon)$ or $V(b+l)-V(b+l-\varepsilon)$ takes a negative value. So, either location $x=b+\varepsilon$ or location $x=b+l-\varepsilon$ gives a higher utility than the equilibrium utility in the cities. This city configuration can therefore not be an equilibrium.

\section{Appendix D: Spatial equilibrium solution}

We define the $n^{s} \times 1$ (column) vector of constants $g^{s}=\left(g_{1}^{s}, \ldots g_{n^{s}}^{s}\right)^{\prime}$ and $f^{s}=\left(f_{1}^{s}, \ldots f_{n^{s}}^{s}\right)^{\prime}$, the $n^{s} \times n^{s}$ matrix of eigenvectors $V^{s}=\left(v_{1}^{s}, v_{2}^{s}, \ldots, v_{N}^{s}\right)$ and the $n^{s} \times n^{s}$ diagonal matrices $C^{s}(x)=\operatorname{diag}\left(\cos \left(\omega_{i}^{s} x\right)\right)$ and $S^{s}(x)=\operatorname{diag}\left(\sin \left(\omega_{i}^{s} x\right)\right)$ where $\operatorname{diag}\left(z_{i}\right)$ is the diagonal matrix with diagonal elements $z_{i}$. The solutions writes in the matrix form as

$$
\mu^{s}(x)=V^{s} C^{s}(x) g^{s}+V^{s} S^{s}(x) f^{s}
$$

Note that $d C^{s}(x) / d x=-W^{s} S^{s}(x)$ and $d S^{s}(x) / d x=W^{s} C^{s}(x)$ where $W^{s}=\operatorname{diag}\left(\omega_{i}^{s}\right)$. Hence, we have that

$$
\dot{\mu}^{s}(x)=-V^{s} W^{s} S^{s}(x) g^{s}+V^{s} W^{s} C^{s}(x) f^{s}
$$

Using $S^{s}(0)=0$ and $C^{s}(0)=I$, we have

$$
\begin{aligned}
& \mu^{s}(0)=V^{s} C^{s}(0) g^{s} \\
& \mu^{s}\left(l^{s}\right)=V^{s} C^{s}\left(l^{s}\right) g^{s}+V^{s} S^{s}\left(l^{s}\right) f^{s} \\
& \dot{\mu}^{s}(0)=V^{s} W^{s} f^{s} \\
& \dot{\mu}^{s}\left(l^{s}\right)=-V^{s} W^{s} S^{s}\left(l^{s}\right) g^{s}+V^{s} W^{s} C^{s}\left(l^{s}\right) f^{s}
\end{aligned}
$$


The border and pasting conditions should determine the $2\left(n^{o}+n^{e}\right)$ constants $g_{k}^{s}$ and $f_{k}^{s}$. Those conditions can be expressed as a system of $2\left(n^{o}+n^{e}\right)$ linear equations:

$$
\begin{aligned}
\mu_{1}^{e}(0) & =\lambda_{A} \\
\mu_{n^{e}}^{e}\left(l^{e}\right) & =\lambda_{A} \\
\mu_{i}^{e}\left(l^{e}\right) & =\mu_{i}^{o}(0), \quad i=1, \ldots, n^{e}-1 \\
\dot{\mu}_{i}^{e}\left(l^{e}\right) & =\dot{\mu}_{i}^{o}(0), \quad i=1, \ldots, n^{e}-1 \\
\mu_{i}^{o}\left(l^{o}\right) & =\mu_{i+1}^{e}(0), \quad i=1, \ldots, n^{o} \\
\dot{\mu}_{i}^{o}\left(l^{o}\right) & =\dot{\mu}_{i+1}^{e}(0), \quad i=1, \ldots, n^{o}
\end{aligned}
$$

Let $\mu=\left(\mu_{1}^{e}, \mu_{1}^{o}, \ldots, \mu_{n}^{e}, \mu_{n}^{o}, \mu_{n+1}^{e}\right)^{\prime}$ be a vector that alternates the elements of the vectors $\mu^{e}(x)$ and $\mu^{o}(x)$ (with $n^{e}+n^{o}=2 n+1$ elements). That is, $\mu=P^{e} \mu^{e}+P^{o} \mu^{o}$ where $P^{e}$ is a $\left(n^{e}+n^{o}\right) \times n^{e}$ matrix such that $P_{i j}^{e}=1$ if $i-1=2(j-1)$ and zero otherwise and where $P^{o}$ is a $\left(n^{e}+n^{o}\right) \times n^{o}$ matrix such that $P_{i j}^{o}=1$ if $i=2(j-1)$ and zero otherwise. For convenience, we denote $\mu(l)=\left(\mu_{1}^{e}\left(l^{e}\right), \mu_{1}^{o}\left(l^{o}\right), \ldots, \mu_{n}^{o}\left(l^{o}\right), \mu_{n+1}^{e}\left(l^{e}\right)\right)^{\prime}$.

We then can write the continuity conditions as

$$
J \mu(l)=L \mu(0)+\lambda_{A} m
$$

where $J$ and $L$ are $\left(n^{o}+n^{e}+1\right) \times\left(n^{o}+n^{e}\right)$ matrices with $J_{i j}=1$ if $i=j+1$ and zero otherwise and $L_{i j}=1$ if $i=j$ and zero otherwise while $m$ is a $\left(n^{o}+n^{e}+1\right)$ vector with $m_{1}=-1, m_{n^{o}+n^{e}+1}=1$ and zero otherwise. To clarify, for $n=2$, this yields the system:

$$
\left[\begin{array}{lllll}
0 & 0 & 0 & 0 & 0 \\
1 & 0 & 0 & 0 & 0 \\
0 & 1 & 0 & 0 & 0 \\
0 & 0 & 1 & 0 & 0 \\
0 & 0 & 0 & 1 & 0 \\
0 & 0 & 0 & 0 & 1
\end{array}\right]\left[\begin{array}{c}
\mu_{1}^{e}\left(l^{e}\right) \\
\mu_{1}^{o}\left(l^{o}\right) \\
\mu_{2}^{e}\left(l^{e}\right) \\
\mu_{2}^{o}\left(l^{o}\right) \\
\mu_{3}^{e}\left(l^{e}\right)
\end{array}\right]=\left[\begin{array}{lllll}
1 & 0 & 0 & 0 & 0 \\
0 & 1 & 0 & 0 & 0 \\
0 & 0 & 1 & 0 & 0 \\
0 & 0 & 0 & 1 & 0 \\
0 & 0 & 0 & 0 & 1 \\
0 & 0 & 0 & 0 & 0
\end{array}\right]\left[\begin{array}{c}
\mu_{1}^{e}(0) \\
\mu_{1}^{o}(0) \\
\mu_{2}^{o}(0) \\
\mu_{2}^{o}(0) \\
\mu_{3}^{e}(0)
\end{array}\right]+\lambda_{A}\left[\begin{array}{c}
-1 \\
0 \\
0 \\
0 \\
1
\end{array}\right]
$$

The pasting conditions for derivatives give

$$
K \dot{\mu}(l)=M \dot{\mu}(0)
$$


where $K$ and $M$ are $\left(n^{e}+n^{o}-1\right) \times\left(n^{e}+n^{o}\right)$ matrix such that $K_{i i}=1$ and zero otherwise and $M_{i j}=1$ if $i-1=j$. For $n=2$, this gives

$$
\left[\begin{array}{ccccc}
1 & 0 & 0 & 0 & 0 \\
0 & 1 & 0 & 0 & 0 \\
0 & 0 & 1 & 0 & 0 \\
0 & 0 & 0 & 1 & 0
\end{array}\right]\left[\begin{array}{c}
\dot{\mu}_{1}^{e}\left(l^{e}\right) \\
\dot{\mu}_{1}^{o}\left(l^{o}\right) \\
\dot{\mu}_{2}^{o}\left(l^{e}\right) \\
\dot{\mu}_{2}^{o}\left(l^{o}\right) \\
\dot{\mu}_{3}\left(l^{e}\right)
\end{array}\right]=\left[\begin{array}{lllll}
0 & 1 & 0 & 0 & 0 \\
0 & 0 & 1 & 0 & 0 \\
0 & 0 & 0 & 1 & 0 \\
0 & 0 & 0 & 0 & 1
\end{array}\right]\left[\begin{array}{c}
\dot{\mu}_{1}^{e}(0) \\
\dot{\mu}_{1}^{o}(0) \\
\dot{\mu}_{2}^{o}(0) \\
\dot{\mu}_{2}^{o}(0) \\
\dot{\mu}_{3}(0)
\end{array}\right]
$$

So, the border and pasting conditions write as the system of $2\left(n^{o}+n^{e}\right)$ equations:

$$
\begin{aligned}
J P^{e} \mu^{e}(l)+J P^{o} \mu^{o}(l) & =L P^{e} \mu^{e}(0)+L P^{o} \mu^{o}(0)+\lambda_{A} m \\
K P^{e} \dot{\mu}^{e}(l)+K P^{o} \dot{\mu}^{o}(l) & =M P^{e} \dot{\mu}^{e}(0)+M P^{o} \dot{\mu}^{o}(0)
\end{aligned}
$$

Plugging the matrix form of $\mu^{s}(x)$, this gives

$$
\begin{gathered}
{\left[\begin{array}{c}
\left(J P^{e} V^{e} C^{e}\left(l^{e}\right)-L P^{e} V^{e} C^{e}(0)\right) g^{e}+\left(J P^{e} V^{e} S^{e}\left(l^{e}\right)-L P^{e} V^{e} S^{e}(0)\right) f^{e} \\
\left(J P^{o} V^{o} C^{o}\left(l^{o}\right)-L P^{o} V^{o} C^{o}(0)\right) g^{o}+\left(J P^{o} V^{o} S^{o}\left(l^{o}\right)-L P^{o} V^{o} S^{o}(0)\right) f^{o}
\end{array}\right]=\lambda_{A} m} \\
{\left[\begin{array}{c}
-\left(K P^{e} V^{e} S^{e}\left(l^{e}\right)-M P^{e} V^{e} S^{e}(0)\right) g^{e}+\left(K P^{e} V^{e} C^{e}\left(l^{e}\right)-M P^{e} V^{e} C^{e}(0)\right) f^{e} \\
-\left(K P^{o} V^{o} S^{o}\left(l^{o}\right)-M P^{o} V^{o} S^{o}(0)\right) g^{o}+\left(K P^{o} V^{o} C^{o}\left(l^{o}\right)-M P^{o} V^{o} C^{o}(0)\right) f^{o}
\end{array}\right]=0}
\end{gathered}
$$

which can be written as

$$
Q\left[\begin{array}{c}
f^{e} \\
g^{e} \\
f^{o} \\
g^{o}
\end{array}\right]=\lambda_{A}\left[\begin{array}{c}
m \\
0
\end{array}\right]=\lambda_{A} q
$$


where using $S^{s}(0)=0$ and $C^{s}(0)=I$

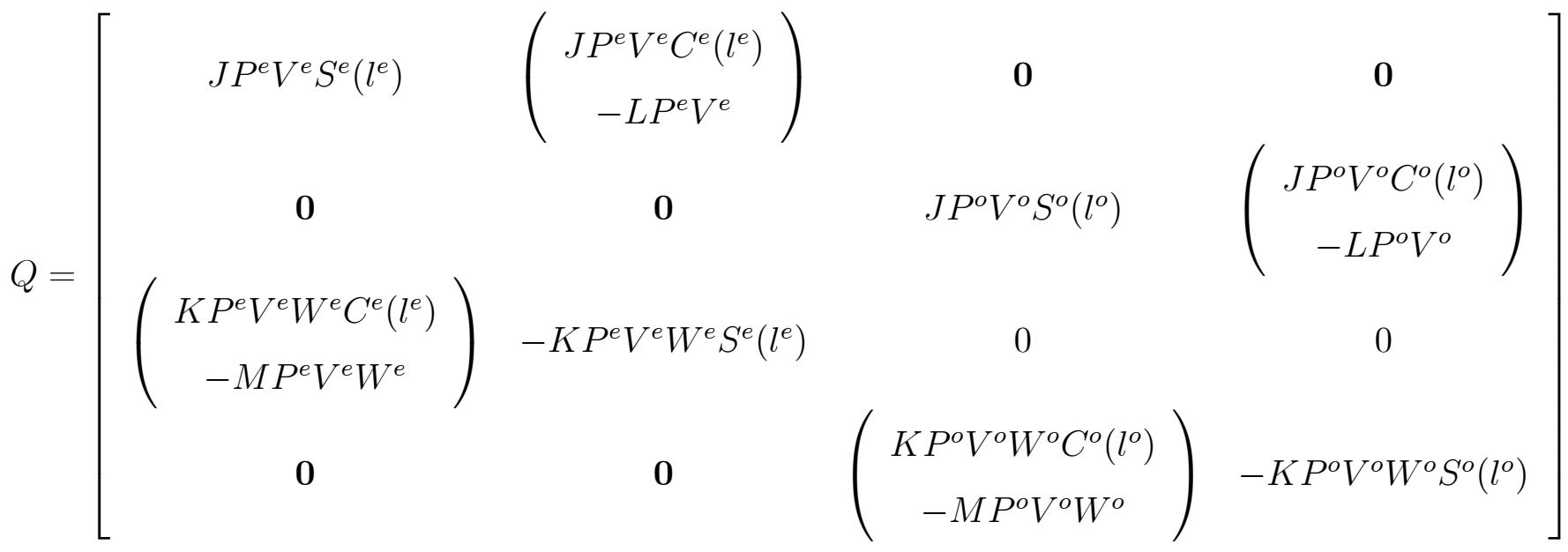

$$
\begin{aligned}
& \text { and } q=\left[\begin{array}{c}
m \\
0
\end{array}\right] \text {. }
\end{aligned}
$$

Let us consider the case of a unique solution ( $\operatorname{det} Q \neq 0$ and $\lambda_{A}>0$ ) where the constants are determined by inverting this system as

$$
f=\lambda_{A} Q^{-1} q
$$

The equilibrium population density then satisfies

$$
\mu^{s}(x)=\lambda_{A} V^{s}\left(C^{s}(x) \quad S^{s}(x)\right) T^{s} Q^{-1} q
$$

where $T^{s}$ is a $N \times 2 N$ matrix that projects the elements of $f$ corresponding to $s$ to the (column) vector $\left(g^{s \prime}, f^{s \prime}\right)^{\prime}$. That is, $\left(g^{s \prime}, f^{s \prime}\right)^{\prime}=T^{s} f .{ }^{1}$ Note that population densities $\mu^{s}(x)$ are proportional to $\lambda_{A}$.

Finally, to get the city population mass, we must integrate all the population density $\mu_{i}^{s}(x)$ on its support $\left[0, l^{s}\right]$ and then aggregate over all supports. Using the fact that $\int^{y} C(y) \mathrm{d} x=\left(W^{s}\right)^{-1} S(y)$ and $\int^{y} S(y) \mathrm{d} x=-\left(W^{s}\right)^{-1} C(y)$ where $\left(W^{s}\right)^{-1}=\operatorname{diag}\left(1 / \omega_{i}^{s}\right)$, the vector of population mass on each interval, $m^{s}=\left(m_{1}^{s}, \ldots, m_{n^{s}}^{s}\right)^{\prime}$ is given by

$$
m^{s}=\int_{0}^{l^{s}} \mu^{s}(x) \mathrm{d} x=\left(\begin{array}{ll}
V^{s}\left(W^{s}\right)^{-1} S\left(l^{s}\right) & -V\left(W^{s}\right)^{-1}\left(C\left(l^{s}\right)-I\right)
\end{array}\right)\left(\begin{array}{c}
g^{s} \\
f^{s}
\end{array}\right)
$$

\footnotetext{
${ }^{1}$ Or equivalently, $T^{e}$ is the Kroeneker delta $\delta_{i, j}$ and $T^{o}$ is $\delta_{i, j+N}$.
} 
where $I$ is the identity matrix. The city population mass $M$ is equal to $\sum_{s \in\{e, o\}} \underline{1}^{\prime} m^{s}$ where $\underline{1}=(1,1, \ldots, 1)^{\prime}$. So,

$$
M=\lambda_{A} \sum_{s \in\{e, o\}} \underline{1}^{\prime}\left(V^{s}\left(W^{s}\right)^{-1} S\left(l^{s}\right) \quad-V\left(W^{s}\right)^{-1}\left(C\left(l^{s}\right)-I\right)\right) T^{s} A^{-1} q
$$

